\title{
Microfluidic-like fabrication of a vanadium-cured bioadhesive by mussels
}

Tobias Priemel ${ }^{1}$, Gurveer Palia ${ }^{1}$, Frank Förste $^{2}$, Franziska Jehle ${ }^{1,3}$, Ioanna Mantouvalou ${ }^{2,4}$, Paul Zaslansky $^{4}$, Luca Bertinetti ${ }^{3}$, Matthew J. Harrington ${ }^{1}$

${ }^{1}$ Dept. of Chemistry, McGill University, 801 Sherbrooke Street West, Montreal, Quebec H3A 0B8, Canada

${ }^{2}$ Dept. of Optics and Atomic Physics, Technische Universität Berlin, Hardenbergstrasse 36, 10623 Berlin, Germany

${ }^{3}$ Dept. of Biomaterials, Max Planck Institute of Colloids and Interfaces, Am Mühlenberg 1, 14476 Potsdam, Germany

${ }^{4}$ Helmholtz Zentrum Berlin, Albert-Einstein-Str. 15, 12489 Berlin, Germany

${ }^{5}$ Dept. for Restorative and Preventive Dentistry, Charité-Universitätsmedizin Berlin, Berlin 14197, Germany

\begin{abstract}
To anchor in seashore habitats, mussels fabricate adhesive byssus fibers mechanically reinforced by protein-metal coordination mediated via 3,4-dihydroxyphenylalanine (DOPA) - providing a well-established role model for bio-inspired design of smart metallopolymers and underwater glues. However, currently, the mechanism by which metal ions are integrated as cross-links during byssus formation is completely unknown. Here, we investigated the byssus formation process, combining traditional and advanced methods to identify how and when metals are incorporated into the material. We discovered that mussels concentrate and store iron and vanadium ions in intracellular metal storage particles (MSPs) complexed with previously unknown catechol-based storage molecules. During thread formation, stockpiled secretory vesicles containing concentrated fluid proteins are mixed with MSPs within a complex microfluidic-like network of interconnected channels where they coalesce forming protein-metal bonds within the nascent byssus. These insights are important for bio-inspired materials design, but also from a biological and chemical perspective - the active accumulation and utilization of vanadium is extremely rare in nature.
\end{abstract}




\section{Introduction}

The mussel byssus is a well-established role model for inspiring the development and fabrication of next generation polymeric materials including self-healing materials, advanced coatings and especially, bio-inspired adhesives $(1,2)$. In particular, the discovery and characterization of protein-metal coordination complexes as dynamic load-bearing cross-links in the byssus (Fig. 1A) (3-5) has led to a surge in development of mussel-inspired metallopolymeric materials $(1,2,6)$. The byssus is a collection of protein-based fibers produced individually by mussels via secretion of more than ten different protein precursors from a specialized organ known as the mussel foot (Fig. 1B) (7-9). At the distal end of the byssal thread is a flattened disc known as the plaque, which can adhere strongly to almost any surface under seawater conditions $(1,8)$. Biochemical and functional investigations have revealed that many of the proteins comprising the plaque are endowed with high concentrations of a post-translational modification known as 3,4dihydroxyphenylalanine (DOPA) in which the side chain of the amino acid tyrosine is converted to a catechol group $(1,8)$. DOPA has the ability to interact with different surface chemistries through various physical and chemical interactions, and thus, plays a crucial role in the adhesion process. Additionally, it was determined that the plaque is cured and mechanically stabilized through the formation of DOPA-metal coordination bonds (Fig. 1A) that provide strong, yet reversible cohesive interactions between the protein building blocks comprising the plaque (10). While it was initially believed that mussels only use iron as a coordinating metal ion, recent evidence suggest that mussels are opportunistic, possibly using other metal ions such as vanadium and aluminum (11-13). In spite of the functional importance of these metal ions, the pathway by which they are introduced into the byssus is currently unknown. Yet, this information is extremely relevant for improving design and fabrication of synthetic mussel-inspired adhesives, coatings and 
metallopolymers, as well as in the rapidly growing field of responsive metallopolymeric materials in general $(1,2,6)$.

It is known that the byssus plaque is formed in just a few minutes from an acidic secretion of fluid protein precursors into a small divot at the tip of the foot on the ventral side known as the distal depression (Fig. 1B-C) $(8,9)$. Prior to formation, the plaque proteins are synthesized and stockpiled within micron-scale secretory vesicles inside cells of a specialized foot gland surrounding the distal depression $(9,14)$. The plaque vesicle contents appear to be highly concentrated and fluid in nature, but during the fabrication process they rapidly cure and crosslink forming a solid open cell porous network $(8,9,15)$. Mature plaques are mechanically stabilized via DOPA-metal coordination cross-links (10); yet, it has been shown that the proteins stored in the plaque vesicles do not exhibit metal coordination, suggesting the metals are introduced at some later point during the formation process $(9,12)$. Early work by Tamarin and colleagues in the 1970s suggested that the plaque vesicles are transported to the distal depression via ciliated channels known as the longitudinal ducts (LDs) that extend from the plaque gland (16). However, since this initial study almost 50 years ago, there is still not a clear understanding of this cellular secretory pathway or the dynamic role of the LDs in in the assembly process - in particular how the fluid plaque proteins mature into a metal cross-linked porous adhesive during their journey along the LDs.

Here, we examined the tissue-based machinery responsible for this remarkable feat of adhesive metallopolymer biomanufacturing and the key role of the longitudinal ducts. In particular, we aimed answer how and when the metal ions are introduced into the nascent plaque material during formation. To achieve this, we investigated the cellular and subcellular structure and composition of the LDs using a combination of methods including light microscopy, electron microscopy, X- 
ray fluorescence microscopy and confocal Raman spectroscopic imaging. Our findings clearly reveal that mussels actively accumulate specific transition metal ions, including iron and vanadium, complexed with an unknown catechol-rich storage molecule within submicron metal storage particles (MSPs) surrounding the LDs. During induced thread formation, the MSPs are cosecreted in a coordinated process into the interconnected network of LD microchannels where they mix with the contents of stockpiled plaque vesicles containing DOPA-enriched plaque proteins. As the mixed MSPs and DOPA-rich proteins proceed toward the distal depression in this microfluidic-like process, the metal ions are released from the MSPs and are coordinated with the plaque proteins, perhaps triggered by a $\mathrm{pH}$ gradient. These findings have important implications both for the understanding of transition metal handling/usage in biology and for fabrication of next-generation bio-inspired metallopolymers and adhesives $(1,2,6)$. In particular, the specific uptake and storage of vanadium is highly unusual and reported in exceedingly few cases in nature, most notably in the blood of ascidians $(17,18)$.

\section{Results}

\section{Anatomy of longitudinal ducts}

MicroCT-tomographic reconstruction of the mussel foot reveals that a complex network of LDs is running through the plaque gland, converging at the distal depression where the plaque is formed (Fig. 1C, Movie S1). This is consistent with the previously proposed role of the LDs as a transport network for the secreted plaque proteins (16). Light microscopy and scanning transmission electron microscopy (STEM) of foot sections reveal that LD microchannels possess a circular cross section between $10-100 \mu \mathrm{m}$ formed by a distinctive columnar epithelial tissue with many cilia and microvilli (Fig. 1D, F). The cilia and microvilli fill almost the entire lumen of the duct, with 
neighboring cilia lining up in the same direction (Fig. 1I), making them practical for transporting materials similar to other ciliated tissue such as bronchial or fallopian tubes (19).

The ducts are surrounded by elongated plaque gland cells containing large amounts of spherical plaque vesicles, which were visualized by Masson's trichrome staining (Fig. 1D). Higher resolution imaging with scanning transmission electron microscopy (STEM) and focused ion beam scanning electron microscopy (FIB-SEM) revealed that cells are densely packed with clusters of these vesicles and that vesicles line up between the epithelial cells ready for secretion (Fig. 1F, H). Reconstruction of the FIB-SEM data further shows the three-dimensional distribution of the vesicles around the LD ready for release into the lumen (Fig. 1I, Movie S2). Spectroscopic analysis with confocal Raman spectroscopy reveals that vesicles contain the plaque proteins identified by their unique Raman spectral fingerprint as described previously (9), which shows a prevalence of the aromatic amino acids tyrosine/DOPA $\left(853,1207\right.$ and $\left.1615 \mathrm{~cm}^{-1}\right)$ and phenylalanine $\left(1003 \mathrm{~cm}^{-}\right.$ 1) (Fig. 1E,J, Fig. S1 and S2).

The epithelial cells surrounding the LD also have a distinctive subcellular structure revealed by STEM and SEM (Fig. 1 G,H), which contains large cell nuclei and many mitochondria. These assignments were confirmed by Raman spectroscopy, which identified cell nuclei by the vibrational peaks of DNA (781 and $1577 \mathrm{~cm}^{-1}$ ) and mitochondria by the resonant peaks of cytochrome c $\left(748,1126\right.$ and $\left.1584 \mathrm{~cm}^{-1}\right)$ (Fig. 1J) (20-23). The large amount of mitochondria are probably necessary to produce the energy needed for active cilia movement and additionally to this, glycogen as a source of energy was detected by Raman spectroscopy based on a strong peak at $482 \mathrm{~cm}^{-1}$ (Fig. 1E,J) $(20,21)$.

As previously reported $(9,12)$, Raman spectra acquired from the plaque secretory vesicles show no evidence of DOPA-metal coordination resonance peaks (Fig. 1J), although this is a prominent 
feature of the native plaque Raman spectrum (10). However, Raman spectroscopic imaging did identify the presence of DOPA-metal coordination outside of the vesicles in tiny submicron spots surrounding the LDs (Fig. 1E,J), as indicated by a highly distinctive set of intense resonant Raman vibrations at 544, 592 and $640 \mathrm{~cm}^{-1}$ (catechol-metal interaction) and $1480 \mathrm{~cm}^{-1}$ (ring vibration) (3, 24). While various metal ions including $\mathrm{V}$ and Fe have been previously detected in Mytilus soft tissue using elemental analysis $(25,26)$, this is the first direct evidence that clearly localizes metal ions within the foot plaque gland, and more specifically surrounding the LDs. Thus, the nature of these particles clearly warrants deeper investigation.
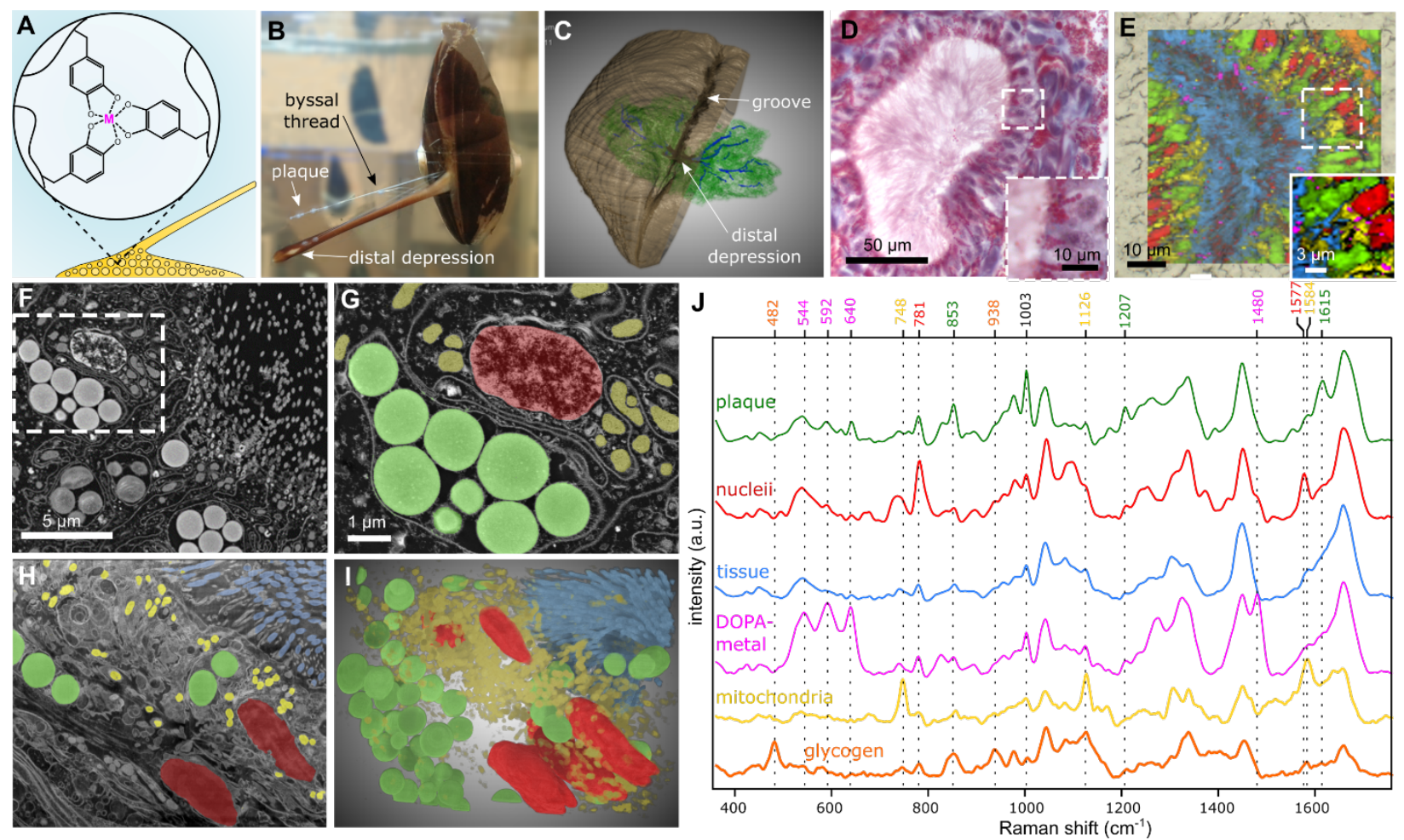

Figure 1: Characterization of the longitudinal ducts found in the mussel foot. (A) Schematic of the DOPA-metal coordination crucial for the mechanical properties of the plaque. (B) Photo of a mussel in the process of byssal thread production with foot fully extended. (C) microCT reconstruction of the distal mussel foot with its plaque gland in green and the longitudinal ducts in blue. (D) Cross section of a longitudinal duct stained with Masson's trichrome. (E) Confocal 
Raman map of an unstained longitudinal duct similar to (C) showing the localization of plaque proteins (green), nuclei (red), cilia (blue), metal storage vesicles (pink), mitochondria (yellow) and glycogen (orange). The colors correspond to spectra in (J) (F) TEM image of part of the longitudinal duct showing cell morphology and cell components. (G) Magnified TEM image from (F) highlighting plaque vesicles in green, cell nucleus in red and mitochondria in yellow (H) FIBSEM image of longitudinal duct highlighting cell components in similar color to (G). (I) 3D reconstruction of the FIB-SEM data from $(\mathrm{H})$. (J) Raman spectra corresponding to cell components shown in (D).

\section{Storage of metal ions}

To further investigate possible storage and secretion of metal ions, we used highly sensitive methods including autometallography (AMG), micro X-ray fluorescence ( $\mu \mathrm{XRF})$ and STEM with energy dispersive X-ray spectroscopy (STEM-EDX). Initial efforts to visualize the metal ions around the LDs with traditional SEM-EDX were not successful (data not shown); however, the detection limit is in the range of $\sim 0.1 \mathrm{wt} \%$ (27). AMG, on the other hand, is an extremely sensitive histological staining method in which heavy metal ions are first transformed into metal sulfides, which then reduce silver ions in the presence of hydroquinone $(28,29)$. Positive AMG staining was observed around the ventral groove (where the thread is formed) and directly around the LDs (Fig. 2A), confirming presence of heavy metal ions in these regions. As AMG is non-specific, $\mu \mathrm{XRF}$ imaging of $200 \mu \mathrm{m}$ thick dried mussel foot sections was performed to determine the identity of the metal ions present around the LDs. Although some impurities were apparent, consecutive sections show clear occurrence of iron and remarkably, vanadium localized around the longitudinal ducts in different mussel feet $(\mathrm{N}=3)$ (Fig. 2B and Fig. S3). Notably, iron was also often localized around the foot groove. 
Further localization of metal at higher spatial resolution was achieved by STEM-EDX of unstained foot sections revealing the presence of vanadium and iron concentrated within submicron-sized particles/vesicles as extremely bright spots (indicating high electron density) surrounding the LDs, which we name here metal storage particles (MSPs) (Fig. 2 C). The MSPs observed with STEMEDX are presumably the same submicron particles detected around the LDs using confocal Raman spectroscopic mapping based on their size and distribution (Fig. 1E, 2D). Furthermore, due to the presence of Raman resonance peaks specific to DOPA-metal coordination (Fig. 2E), these findings indicate that the metals in the MSPs are very likely coordinated within an organic complex that contains catechol groups. This is further supported by the detection of nitrogen, oxygen and carbon in the MSPs by STEM-EDX (Fig. 2C, Fig. S4). The metals can be easily removed via EDTA treatment, resulting in the disappearance of the characteristic DOPA-metal resonance Raman peaks, demonstrating the ability of the MSPs to exchange metal ions with other ligands (Fig. S5). Importantly, brief treatment of EDTA treated sections with a $1 \mathrm{mM} \mathrm{VCl}_{3}$ solution led to the appearance of DOPA-V resonance Raman peaks not only in the MSPs, but also throughout the entire section localized within the plaque vesicles rather than in small, localized hotspots. This clearly indicates: 1) that the MSPs are highly effective in keeping the metals separate from the plaque proteins prior to secretion and 2) that the plaque vesicle proteins strongly coordinate vanadium when exposed (Fig. S5). Notably, the coordination complexes in the MSPs seem to vary slightly from particle to particle, since peak position and shape of the resonance Raman bands differ between different MSPs (Fig. S6), which may indicate coordination of different metals, differences in coordination bond length or slight variations in coordination geometry $(3,30)$. Indeed, the storage of mixtures of vanadium and iron in different ratios may partially explain the peak position diversity, which is also consistent with the STEM-EDX and $\mu \mathrm{XRF}$ findings. This is 
also consistent with previous reports that mussels are typically opportunistic with the metals that are used to fortify the byssus depending on what is available in their local environment (11).

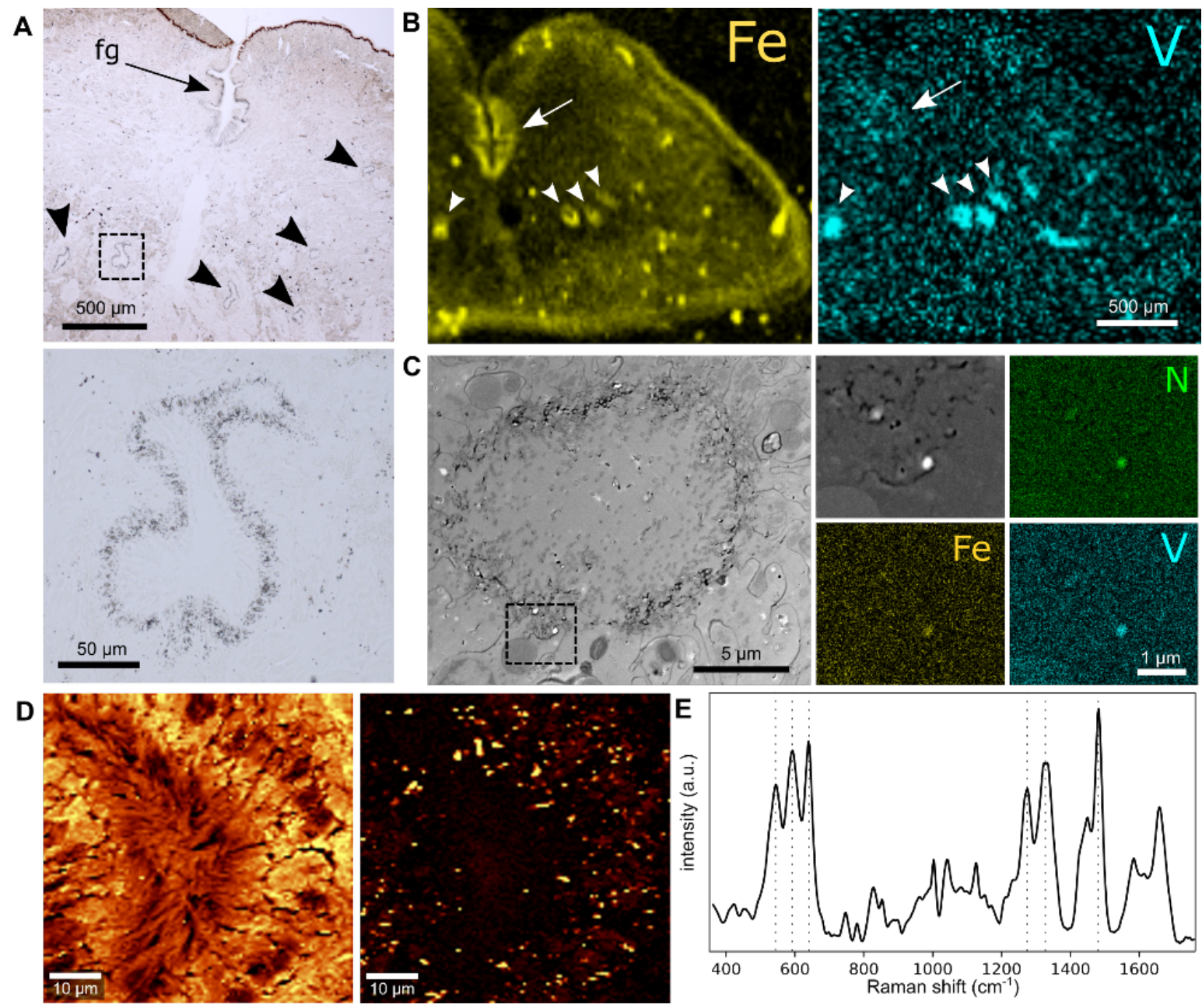

Figure 2: Metal storage around the longitudinal ducts. (A) Autometallography staining of a mussel foot cross section with positive staining around the LDs (arrowheads) and around the mussel foot groove (fg). Dotted box indicates the magnified image in the lower panel (B) $\mu \mathrm{XRF}$ measurements on $500 \mu \mathrm{m}$ thick mussel foot cross sections showing the distribution of iron (yellow) and vanadium (turquoise) with arrow heads indicating the LDs and an arrow indicating the groove. (C) STEM-EDX analysis of metal storage vesicles around a LD showing distribution of nitrogen in green, iron in yellow and vanadium in turquoise (D) Raman maps of LD corresponding to the $\mathrm{CH}$-stretch (2900-3000 $\left.\mathrm{cm}^{-1}\right)$ on the left side and corresponding to the spectrum in (E) on the right 
side. (E) Raman spectrum of the metal storage vesicles showing DOPA-metal coordination resonance peaks highlighted by dotted lines.

\section{Secretion of proteins and metal ions via the longitudinal ducts}

Thus far, our study of the LDs paints a picture of a complex network of interconnected microchannels surrounded by the stockpiled building blocks (proteins and metals) required to build the plaque and equipped with the cilia needed to transport this cargo to its destination at the distal depression. Yet, this picture is static. In order to understand how these various parts work together, it is necessary to follow the dynamic plaque formation process. To study this process and the role the LDs, we induced the plaque secretion process by an injection of $\mathrm{KCl}$ solution into the pedal ganglion at the base of the foot as described previously $(9,16)$. We then froze the secretion process at various time-points and analyzed the tissue using histological imaging, FIB-SEM and confocal Raman spectroscopic imaging.

Histological imaging of induced feet shows that plaque vesicles are secreted into the LDs where they coalesce into a condensed fluid mass that stains consistently and homogenously and that increases in size closer to the distal depression (Fig. 3A). Raman spectroscopic imaging of the coalescing mass in the LD lumen at early stages of secretion indicates a homogenous composition with a spectrum nearly identical to that observed in the plaque vesicles. FIB-SEM reconstruction reveals a more detailed snapshot of the formation process with numerous vesicles being secreted through the cilia and mixing together in the lumen (Fig. 3C, Movie S3). In the nascent induced plaque, a number of vesicles can be observed intact, while the contents of other vesicles are coalesced into a fluid mass as described above, with some evidence of nanoporosity observed, reminiscent of the porous native plaque structure (15). 

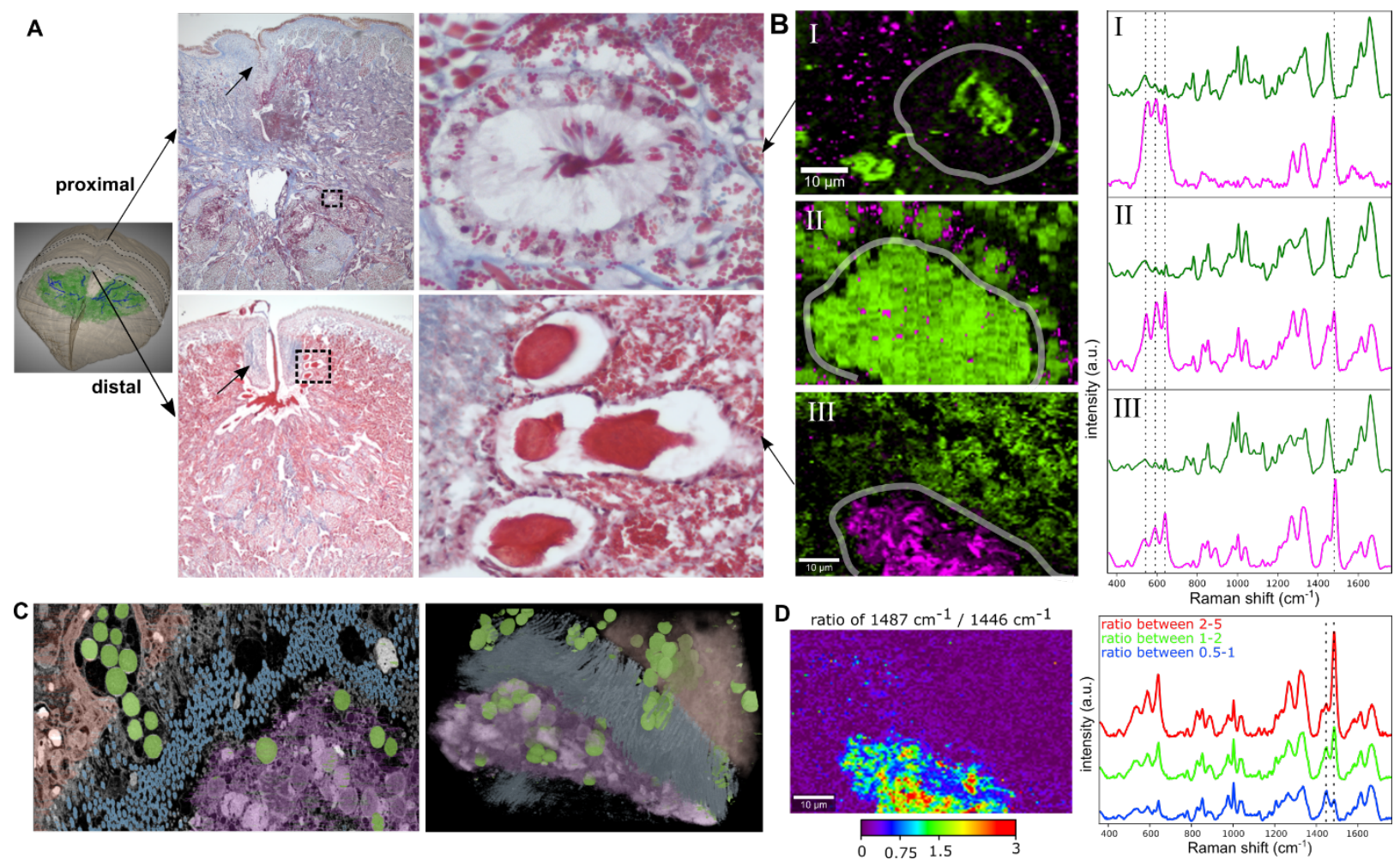

Figure 3: Secretion of the plaque proteins and metal-ions. (A) Masson's trichrome staining of mussel foot cross section after induction of secretion. Magnified images on the right side show how plaque proteins are secreted into the ducts (B) Raman image maps showing the distribution of plaque proteins in green and DOPA-metal coordination in pink (boundary of LD highlighted with white line) at different stages of the secretion process (I-III indicate increasing proximity to the distal depression). Corresponding average Raman spectra are provided on the right side. (C) FIB-SEM analysis of induced LD showing secretion of plaque vesicles (green) into the lumen of the LD where they coalesce into the nascent plaque (highlighted in purple). Cilia (blue) all line up in the direction of secretion. 2D image is provided on the left and the $3 \mathrm{D}$ reconstruction is on the right. (D) Raman spectral analysis of the induced LD showing the distribution of coordinated metal ions via the ratio of DOPA-metal resonance peak $\left(1487 \mathrm{~cm}^{-1}\right)$ to a protein peak $\left(1446 \mathrm{~cm}^{-1}\right)$. Low concentrations are highlighted with cold colors and high concentrations in warm colors. Corresponding Raman spectra (averages of different ratios) on the right side. 
In addition to the protein secretion, Raman imaging provided insights into the secretion process of the MSPs and their mixing with the plaque proteins (Fig. 3B). While artificial thread induction is not a finely controlled process, we were able to observe different stages of this secretion process that appear to be related to the time after induction and position within the LD (i.e. closer to or further from the distal depression). At early stages of secretion, distant from the distal depression, we observed primarily plaque proteins present in the ducts as described above, whereas the MSPs are still observed in the surrounding tissue. At later stages closer to the distal depression, the MSPs appear to be also secreted into the lumen of the duct and mixed with the protein (Fig. 3B); however, at this stage, the metals are still localized in submicron spots of the image and do not appear to bind to the surrounding plaque proteins. Finally, near the distal depression, DOPA-metal coordination was detected via Raman spectroscopy throughout the majority of the dense protein phase in the lumen, ostensibly due to mixing and diffusion of metal ions from the MSPs (Fig. 3B). In support of this, the intensity of the DOPA-metal resonance Raman peaks relative to the protein peaks vary in different regions of the nascent plaque with the appearance of hotspots in which intensity of metal coordination decreases gradually, moving away from the hotspot (Fig. 3D).

\section{Discussion}

The current study provides several important insights into the plaque formation process relevant to our biological understanding and its potential for bio-inspiration: 1) The metal ions used to form the plaque are actively accumulated by mussels and stored in the foot separately from plaque proteins in small particles complexed with a catechol containing molecule. 2) Vanadium, in particular, is accumulated and used by mussels for plaque formation, which is striking given the rarity of this metal in functional roles in biology. This raises many interesting questions of how $\mathrm{V}$ is acquired and stored, and why it is being used. 3) Plaque formation proceeds through a 
microfluidic-like process in which stockpiled precursor proteins and MSPs are deployed, transported and actively mixed through a multiplex network of cell-lined microchannels. 4) As the secreted contents move through the microchannels, the MSPs release their metal ions (perhaps through a $\mathrm{pH}$-driven process), which then diffuse into and spread throughout the nascent plaque where they are coordinated by DOPA residues in the plaque proteins. Previous work show this provides cohesive interactions that facilitate the curing of the fluid into a solid adhesive under seawater conditions $(10)$.

The discovery of the MSPs solves a long-standing mystery as it was previously unresolved how metal ions were added to the byssus - passively acquired from seawater or actively added by the mussel. Previous studies showed that metal ions could be removed from threads via EDTA chelation, and then replaced by soaking threads in millimolar metal chloride solutions, supporting the passive hypothesis $(3,11)$. However, it is important to mention that seawater concentrations of soluble Fe and $\mathrm{V}$ are in the range of $1-50 \mathrm{nM}(31,32)$. In support of the active uptake hypothesis, mussels are filter feeders that accumulate a broad range of metals present in the water column within both their soft tissue as well as their byssus (including $\mathrm{Ag}, \mathrm{Cd}, \mathrm{Cr}, \mathrm{Cu}, \mathrm{Fe}, \mathrm{Ni}, \mathrm{Pb}, \mathrm{Ti}, \mathrm{V}$ and $\mathrm{Zn}$ ), which led to the use of the byssus as heavy metal pollution biomarkers since the 1970s $(25,33-35)$. Previously, an iron radiolabeling study has provided the only strong evidence that the metals that end up in the byssus are first actively accumulated by the mussel in the soft tissue, although it stopped short of localizing iron storage specifically in the foot (36). Many invertebrates possess metal-containing granules (37) and membrane bound metal particles containing $\mathrm{Zn}, \mathrm{Fe}$ and $\mathrm{Ca}$ were previously observed and isolated from the kidney tissue in M. edulis (38). The current discovery of $\mathrm{V}$ and Fe in the submicron-sized metal storage particles (MSPs) and their co-secretion and mixing/cross-linking with the plaque proteins provides clear evidence that mussels leave 
nothing to chance, given the extremely low concentrations of these metals in seawater (31) and their crucial role in byssus mechanics $(10,11)$.

Throughout biology, physiologically relevant metal ions (e.g. Fe, $\mathrm{Zn}, \mathrm{Cu}$ ) are almost always taken up, stored, transported and regulated in living organisms in close association with biomolecules (e.g. ferritin, transferrin, metallothionein) $(31,33)$. Similarly, we infer that storage of iron and vanadium in the MSPs separately from the plaque proteins complexed with a catechol-based storage molecule confers several advantages. For example, this precaution almost certainly circumvents premature cross-linking of the plaque proteins either via metal cross-linking at neutral to basic $\mathrm{pH}(9,39,40)$ or covalent oxidative cross-linking at low $\mathrm{pH}(41)$ that would be expected to occur if they were not separated. The complexation of Fe and V during storage is also likely important since both are highly redox active and could potentially mediate unwanted side reactions in the cellular environment leading to the production of harmful reactive oxygen species (ROS) (42). Additionally, complexation with catechols has been seen to influence the redox state of $\mathrm{V}$, as had been seen in both biomolecular and synthetic systems (43-45). Based on our current findings, it is unclear if the metals are stored as individually coordinated ions or perhaps as ion clusters or an amorphous mineral phase (as in ferritin). Nonetheless, our findings indicate they are effectively partitioned from the plaque proteins.

Of particular relevance, the mussel's storage and use of vanadium to fabricate an adhesive material is remarkable considering there are only three other examples of organisms known to accumulate V to a significant degree - ascidians, the mushroom species Amanita muscaria and some species of seballarid polychaetes $(43,45,46)$. Notably, the function of $\mathrm{V}$ is not well established in any of these three examples, even after more than 100 years of study in the case of ascidians $(43,46)$, whereas the current study and others indicate that $\mathrm{V}$ is serving a clear mechanical cross-linking 
function in the byssus plaque $(10)$, as well as the byssus cuticle $(11,13)$, which has been mimicked successfully in mussel-inspired hydrogels (39). The most prominent and well-studied example of V hyperaccumulation is in ascidians (a.k.a. tunicates or sea squirts) $(17,43,47)$, which can reach a concentration of up to $\sim 350 \mathrm{mM}$ in the blood of some species, which is 10 million-fold higher than the seawater concentration (48). This raises the question of how vanadium is specifically concentrated in biology, including by mussels. In seawater, almost all the vanadium is present primarily in the monomeric pentavalent ionic form $\mathrm{H}_{2} \mathrm{VO}_{4}{ }^{-}$, which is similar in structure to a phosphate anion, leading to the proposal that it might be initially acquired by organisms through existing phosphate uptake pathways $(31,43)$. However, in ascidians, this $\mathrm{V}(\mathrm{V})$ form is reduced to V(IV) and V(III), both of which are unstable at physiological $\mathrm{pH}$. To stabilize these oxidation states, it was shown that $\mathrm{V}(\mathrm{IV})$, which is present in the cytoplasm of ascidians is complexed by a Cys-rich protein known as vanabin via interaction with Lys, Arg and His residues $(18,32)$, which is proposed to function both as a transporter and reducing agent. V(III) in ascidians, on the other hand, is heavily concentrated and stabilized within small cells in the blood known as vanadocytes in which the V(III) is associated with high concentrations of sulfate ions and held at an extremely low $\mathrm{pH}(0-3)$, under which the $\mathrm{V}(\mathrm{III})$ form is stabilized $(43,48)$. In the mushroom A. muscaria, hyperaccumulated V ions are stored in the V(IV) oxidation state associated with a small molecule natural product known as amavadin; yet, similar to ascidians, the function of $\mathrm{V}$ remains elusive $(31,43,45)$.

Our findings indicate that the storage of $\mathrm{V}$ (and Fe) in the MSPs in the mussel foot is mediated by a different mechanism than that observed in either ascidians or $A$. muscaria. Indeed, the metal ions stored in the MSPs are complexed via catechol moieties, which is previously unobserved in nature, as far as we are aware. While we do not know the nature of the organic molecule or the internal 
conditions of the MSPs, we infer that the conditions are not acidic due to the presence of the charge transfer peak in the resonance Raman spectrum $\left(\sim 541 \mathrm{~cm}^{-1}\right.$, Fig. 2E, S5) indicating bidentate chelation (24), which is not favored at acidic $\mathrm{pH}(39,40)$. Tunicates also contain a molecule known as tunichrome that contains pyrogallol moieties, structurally and functionally similar to catechols, that are capable of coordinating $\mathrm{V}$ ions and reducing $\mathrm{V}(\mathrm{V})$ to $\mathrm{V}(\mathrm{IV})$; however, it is now accepted that tunichrome and $\mathrm{V}$ are stored separately in the ascidian blood and do not interact in vivo (43, 45)). Similarly, it was shown that the bacterial siderophore enterobactin, which possesses three catechol groups, is able to complex V(IV), although its biological function is to scavenge $\mathrm{Fe}(\mathrm{III})$ in iron-limited marine environments (49). Thus, the unknown organic molecule within the MSPs appears to be the first reported instance of catechol-based molecule functioning to store and transport $\mathrm{V}$, as well as $\mathrm{Fe}$, in a biological role in vivo. It seems plausible that the $\mathrm{V}$ is stored in the $\mathrm{V}(\mathrm{IV})$ oxidation state since catechols have been previously shown reduce $\mathrm{V}(\mathrm{V})$ to $\mathrm{V}(\mathrm{IV})$ following complexation $(43,50)$. Consistent with this, V(IV) was previously found to be present in native thread cuticle and plaque materials using EPR (11). However, the exact oxidation state in the thread and during storage remains to be verified.

An important open question concerns why the mussel is using $\mathrm{V}$ in the first place - what are the functional advantages? It was recently observed in the mussel byssus cuticle, which provides a hard coating on the byssus fibers, that vanadium is co-localized and coordinated to DOPA-rich proteins in hard submicron granules (13) whereas iron was observed to self-segregate and colocalize with sulfur-rich matrix proteins. It was proposed in this case that this metal self-sorting is a diffusion and competition effect related to the longer bond lifetime (i.e. slower dissociation kinetics) of the DOPA-V vis-a-vis the DOPA-Fe complexes (39). Notably, this kinetic difference manifested as a more solid-like behavior in viscoelastic catechol-based hydrogels based on V vs. 
Fe (39). Analogously, metal storage using a catechol-based molecule may have a natural preference to concentrate $\mathrm{V}$ over Fe for kinetic reasons, although the exact mix of metals may depend strongly on the local water chemistry. Indeed, there is evidence that mussels are opportunistic regarding which metal ions they acquire and utilize to construct their byssus depending on the local composition of the seawater $(5,34,51)$. Nonetheless, there is growing evidence, primarily based on reassessment of resonance Raman signals from this and earlier studies, that DOAP-V coordination is a common feature as observed in the byssal threads of at least three different mussel species from the Mytilus genus acquired from very different bodies of water including the coast of California, the Mediterranean Sea and the Atlantic Ocean off the coast of Canada (Table S1, Fig. S7) $(3,9,10,12)$. This suggests some preference to use vanadium, which may reflect the catechol chemistry used to store the metals.

\section{Model of plaque formation}

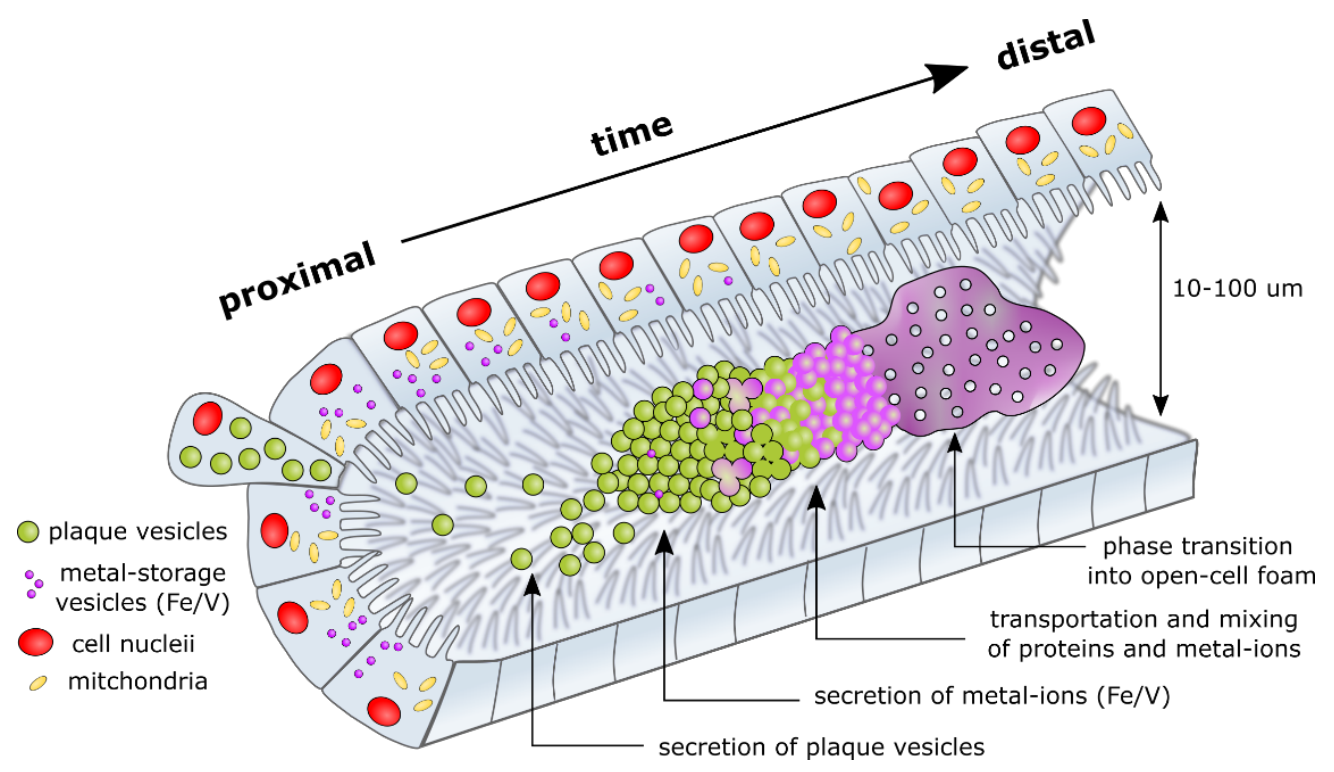

Figure 4: Illustrative model of the secretion process during plaque formation. During plaque formation, the mussel secretes plaque vesicles and MSPs into the lumen of the LD microchannels where they are transported and mixed by active cilia movement. The Fe and V metal ions diffuse 
out of the MSPs and distribute throughout the dense protein phase eventually forming $\mathrm{pH}-$ dependent DOPA metal coordination bonds that mechanically fortify the porous plaque structure once it is released into seawater.

This investigation also provides important insights into the microfluidic-like machinery used to fabricate the plaque adhesive and the spatiotemporal control of plaque assembly that in light of previous studies, allows us to posit an updated model of plaque formation (Fig. 4). During the formation process, plaque protein vesicles are secreted into the LDs where the contents of multiple vesicles coalesce, forming a dense phase that can flow as a fluid through the ducts toward the distal depression. At a later point, the Fe- and V-enriched MSPs are secreted into the lumen of the LDs, resulting in a very localized metal distribution in the nascent plaque material. Through further mixing of the plaque proteins and MSPs, likely facilitated via active cilia movement and diffusion, the metal ions spread throughout the complete protein phase. The relative coordination strength of the catechols in the MSPs vis-à-vis the plaque protein DOPA residues for the metal ions may be influenced by physicochemical conditions within the LDs (e.g. pH, salt content or possibly chemical differences in the MSP catechols that their lower binding affinity). Indeed, it is known that the plaque proteins are stored and secreted at a $\mathrm{pH}$ as low as 2 (52) where coordination of metal ions via catechols is quite weak. In contrast, as already mentioned, the observed resonance Raman signal of the MSPs clearly indicates bidentate chelation by DOPA (24), which is typically only observed at elevated $\mathrm{pH}$ values $(39,40)$.

Given these observations, one compelling hypothesis is that when the comparatively small MSPs are exposed to the acidic conditions of the larger fluid plaque secretion, the affinity of the catechols within the MSPs for the metal ions is reduced, leading to metal release and diffusion throughout the liquid protein phase as it is secreted, further enhanced by cilia-driven mixing. As the $\mathrm{pH}$ is 
increased to the slightly basic $\mathrm{pH}$ of seawater $(\sim \mathrm{pH} 8)$, the dispersed metal ions are again chelated by DOPA-enriched plaque proteins, such as $\mathrm{mfp}-2$, forming cohesive coordination bonds that mechanically fortify the plaque structure. Previous findings are consistent with this sequence of events. For example, it was proposed that during plaque curing, the nascent plaque proteins undergo a phase transition from a fluid phase forming a micro- and nanoporous solid $(9,15)$. It is so far unknown if this process is also mainly triggered by the change in $\mathrm{pH}$, addition of metals or if other changes in the physicochemical environment lead to this structural change (e.g. change in ionic strength, presence of other ions, redox conditions). However, it was recently shown that freshly formed plaques placed immediately in acidic conditions do not form pores and are much softer (53), consistent also with the $\mathrm{pH}$-dependent nature of the metal coordination process.

Overall, plaque formation seems to be a complex orchestrated exocrine secretion process that is regulated by both physicochemical (change of $\mathrm{pH}$ ) and biological (active cilia movement) driving forces. The insights from this study are especially relevant to the growing field of mussel-inspired polymers, but also to the broader field of sustainable polymer production or even tissue engineering. For example, the prestorage of metal ions as complexed particles for gradual release may present a more effective means of mixing, which has remained a key processing challenge since the very first mussel-inspired metallopolymers were designed $(2,39,40)$. More generally, understanding how the physicochemical environment can trigger a fluid-to-solid phase transformation may inspire the development of novel 3D printing techniques operating under environmentally friendly conditions. In particular, the spontaneous formation of a hierarchically porous solid from a liquid precursor is remarkable and a deeper understanding of the physicochemical driving forces guiding this process is needed. 


\section{Acknowledgements}

This work was supported by the Natural Sciences and Engineering Research Council of Canada (NSERC Discovery Grant RGPIN-2018-05243) and a Canada Research Chair award (CRC Tier 2 950-231953) and the Max Planck Society. TP was funded partially by a FQRNT Quebec Merit Fellowship for Foreign Students. The authors thank B. Kanngießer for helpful discussion and insight, S. Sviben for providing training on sample preparation methods (high pressure freezing), C. Schmitt for providing Raman spectra of M. galloprovincialis threads and the staff at FEMR (McGill) for their support in imaging.

\section{Methods}

\section{Sample preparation}

Mytilus edulis mussels farmed on Prince Edward Island were kept in an aquarium with artificial seawater (Fluval Sea, USA; containing $\mathrm{Ca}, \mathrm{K}, \mathrm{Mg}$ and $\mathrm{Sr}$ ) adjusted to $3 \%$ at $15{ }^{\circ} \mathrm{C}$. Mussel feet were dissected from adult mussels and either fixed in 4\% paraformaldehyde (PFA) overnight or directly frozen in OCT medium with liquid nitrogen-cooled isopentane. These frozen samples were kept frozen at $-80{ }^{\circ} \mathrm{C}$ and prior to investigations, sectioned with a cryotome (Leica) at $-25{ }^{\circ} \mathrm{C}$ into $5 \mu \mathrm{m}$ sections for histological staining, AMG and Raman spectroscopy and into $200 \mu \mathrm{m}$ sections for XRF investigations. To study the secretion process, live mussels were opened and injected with a $0.56 \mathrm{M}$ potassium chloride solution in the base of the foot, as described previously (16). The mussel foot was then excised after 5-10 min and directly frozen in OCT medium with liquid nitrogen-cooled isopentane. These induced mussel foot samples were handled in the same way as described already for the uninduced mussel feet. 


\section{Micro computed tomography (CT)}

Mussel feet for microCT were fixed in $4 \%$ PFA overnight and afterwards washed with PBS buffer. The fixed foot was then stained overnight in Lugol's solution followed by a brief wash with water. The foot was mounted upright in a PVA vial under $100 \%$ relative humidity $(\% \mathrm{RH})$ and scanned in a Bruker MicroCT (1172, Skyscan, Kontich, Belgium) using $60 \mathrm{kV}$ source energy, an effective pixel size of $2.5 \mu \mathrm{m}$ and an angular increment of $0.15^{\circ}$. Data were reconstructed by the filterback projection method using the manufacturer software nRecon (v. 1.6.10.4, Bruker-microCt, Kontich, Belgium), then imaged and segmented using Amira (Amira ZIB edition 2016.14, Zuse Institute Berlin). The segmented data was transferred to Dragonfly 4.1 (Object Research Systems, Montreal, Canada) for 3D visualization and animation.

\section{Masson's Trichrome staining}

Masson's Trichrome staining (Thermofisher) was performed according to manufacturer's protocol as described previously (9). In short, $5 \mu \mathrm{m}$ cryo-sections were fixed in Bouin's solution overnight, rinsed with tap water and subsequently stained in Weigert's iron hematoxylin, Bieberich scarletacid fuchsin, phosphomolybdic-phosphotungstic acid and aniline blue solution each for $5 \mathrm{~min}$. Afterwards, the sections were differentiated in $1 \%$ acetic acid for $5 \mathrm{~min}$, washed with distilled water and dehydrated through an ascending alcohol series. Finally, sections were cleared in NeoClear (VWR) and mounted in NeoMount (VWR).

\section{Autometallography}

In order to find and locate heavy metal ions in the mussel foot, Danscher's staining method called Autometallography (AMG) $(28,29)$ was used, which is based on the sulphide silver method of Timm from 1958 (54). Dissected mussel feet were fixed in 3\% glutaraldehyde for $5 \mathrm{~h}$, washed with PBS and incubated overnight in 1\% sodium sulfide buffered with PBS (pH 7.5). Mussel feet 
were then washed several times in PBS, frozen in OCT medium and $5 \mu \mathrm{m}$ cryo-sections were cut. Sections were stained in a freshly prepared staining solution for 20-40 min in the dark. The staining solution consisted of $0.2 \mathrm{M}$ citrate buffer, $0.3 \mathrm{~g} / \mathrm{ml}$ gum arabic, $8.5 \mathrm{mg} / \mathrm{ml}$ hydroquinone and 1.2 $\mathrm{mg} / \mathrm{ml}$ silver nitrate. After development, sections were washed with water, dehydrated in alcohol and mounted with NeoMount.

\section{Confocal Raman spectroscopy}

For Raman spectroscopy, unfixed mussel feet were cryo-sectioned into $5 \mu \mathrm{m}$-thick sections and mounted on glass slides. Raman spectra were collected using a confocal Raman microscope (Alpha 300R, WITec, Germany) equipped with a green laser $(532 \mathrm{~nm})$. The laser at a power of $5 \mathrm{~mW}$ was focused with a 100x objective (Zeiss, numerical aperture $[\mathrm{NA}]=0.9$ ) and the scattered light was collected with a thermoelectrically cooled CCD detector behind a $600-\mathrm{g} / \mathrm{mm}$ grating at a spectral resolution of $4 \mathrm{~cm}^{-1}$. Image scans were performed in the TrueSurface mode of the instrument collecting 1, 2 or 3 spectra per micrometer at an integration time of $5 \mathrm{~s}$ per pixel. WITec's ControlFIVE and ProjectFIVE plus software were used to collect and process the Raman spectra, respectively. For spectral processing, cosmic rays were removed first, followed by a background subtraction. Characteristic spectra for different regions were identified using linear combinations of single spectra and these spectra were averaged and smoothed.

\section{micro X-ray Fluorescence $(\mu \mathrm{XRF})$}

$\mu \mathrm{XRF}$ scans on 26 sections ( $200 \mu \mathrm{m}$ thick) acquired from three different mussel feet were conducted. Sections were prepared on carbon tape and measurements performed with a modified commercial $\mu \mathrm{XRF}$ instrument (Bruker M4 tornado) (55), which enables measurements in $\mu \mathrm{XRF}$ and confocal $\mu \mathrm{XRF}$ mode by switching between a detector with and without an attached X-ray optic. Experiments were conducted in ambient atmosphere with the tube current and voltage of the 
Rh micro-focus tube set to $600 \mu \mathrm{A}$ and $50 \mathrm{kV}$, respectively. No filter was used. Measurement times were $3 \mathrm{~s}$ to $15 \mathrm{~s}$ per pixel collected in a cyclic mode with several hundreds of cycles per image. The elemental images were extracted from the manufacturer's software as 16-bit tiff images using the fast deconvolution setting and the final graphics prepared with ImageJ.

\section{Cryo-/chemical fixation and embedding}

Mussel foot tissue samples for electron microscopy were either cryo-fixed or chemically fixed. For cryo-fixation, a dissected foot was cut into transverse sections around the distal depression and very small pieces of plaque gland tissue were transferred into hexadecane-filled carriers and highpressure frozen in a Leica HPM 100. Freeze substitution was carried out in a Leica EM AFS-2 at $-85{ }^{\circ} \mathrm{C}$ for $103 \mathrm{~h}$ and fixation (in $0.1 \% \mathrm{OsO} 4,0.1 \%$ Uranyl acetate, $0.5 \%$ glutaraldehyde, $1.5 \%$ $\mathrm{dH}_{2} \mathrm{O}$ and acetone) at $-20{ }^{\circ} \mathrm{C}$ for $12 \mathrm{~h}$. Samples were brought to RT and washed $5 \mathrm{x}$ in $100 \%$ acetone (EM grade). For chemical fixation, dissected feet were blotted with a paper towel to remove mucus and pre-fixed for $30 \mathrm{~min}$ at $4{ }^{\circ} \mathrm{C}$ in $3 \%$ glutaraldehyde, $1.5 \%$ paraformaldehyde, $650 \mathrm{mM}$ sucrose in $0.1 \mathrm{M}$ cacodylate buffer $\mathrm{pH}$ 7.2. The foot tissue was then cut into thin crosssections comprising the groove and part of the gland tissue and fixed for $2 \mathrm{~h}$ at $4{ }^{\circ} \mathrm{C}$ in the same buffer as above. Fixed samples were rinsed $5 \times$ with $0.1 \mathrm{M}$ cacodylate buffer, $\mathrm{pH} 7.2$ at $4{ }^{\circ} \mathrm{C}$ and post-fixed with $1 \% \mathrm{OsO}_{4}$ for $1 \mathrm{~h}$ at $4{ }^{\circ} \mathrm{C}$. Tissue samples prepared for elemental analysis were not treated with $\mathrm{OsO}_{4}$. Samples were rinsed again in $\mathrm{dH}_{2} \mathrm{O}\left(3 \times 5\right.$ min at $\left.4{ }^{\circ} \mathrm{C}\right)$, followed by series dehydration in acetone $(50 \%, 70 \%, 90 \%, 3 \times 100 \%)$ for 10 min each at RT. Cryo-fixed and chemically fixed samples were embedded in Epoxy (Epon 812 substitute, Sigma-Aldrich, \# 45359) for FIB-SEM $(\mathrm{N}=3)$ and STEM $(\mathrm{N}=3)$ and polymerized at $70{ }^{\circ} \mathrm{C}$ for at least $48 \mathrm{~h}$. Ultrathin sections of $100 \mathrm{~nm}$ for STEM investigations were prepared using an ultramicrotome and mounted on carbon coated $\mathrm{Cu}$ grids (200 mesh). 


\section{STEM/STEM-EDX}

Scanning transmission electron microscopy (STEM) was performed with a Thermo Scientific Talos F200X G2 S/TEM equipped with a X-FEG High Brightness Schottky Field Emission Source and a Ceta 16M CMOS Camera, operated at $200 \mathrm{kV}$ acceleration voltage. STEM mode (camera length $98 \mathrm{~mm}$, collection angle 58-200 mrad) was used for energy dispersive X-ray spectroscopy (EDX) and high angle annular dark field (HAADF) imaging. In STEM mode, a fine electron probe scans the surface of the sample pixel-by-pixel enabling identification of the area of the sample that generates certain characteristic X-rays with nanometric resolution, which were detected with a Super-X Windowless Energy Dispersive Spectrometer (Four quadrant in-column SDD detector). STEM elemental maps (intensity) were recorded at the region of interest for at least $30 \mathrm{~min}$.

\section{FIB-SEM sample preparation and measurements}

Resin blocks containing foot tissue samples $(\mathrm{N}=3)$ were polished in order to expose the tissue at the block surface. Samples were sputter-coated with three carbon layers $(\sim 5 \mathrm{~nm}$ each) and one platinum layer $(\sim 5-10 \mathrm{~nm})$ and transferred to the Zeiss Crossbeam 540 (Carl Zeiss Microscopy GmbH, Germany). At the region of interest, a trench for SEM imaging was milled into the sample surface using a current of $65 \mathrm{nA}$ at $30 \mathrm{kV}$ acceleration voltage. The resulting cross-section was finely polished using the $3 \mathrm{nA}$ FIB probe at $30 \mathrm{kV}$. Thin slices of samples were removed in a serial manner by FIB milling (either $1.5 \mathrm{nA}, 30 \mathrm{kV}$, slice thickness $12 \mathrm{~nm}$ or $700 \mathrm{pA}, 30 \mathrm{kV}$, slice thickness $20 \mathrm{~nm}$ ). After each milling step, the specimen was imaged by SEM (acceleration voltage $=2 \mathrm{kV}$ ) using the secondary electron (SE) and backscattered electron detector (BSE). The image resolution was $1024 \times 756$ pixels with a lateral image pixel size of either $14.78 \mathrm{~nm}$ or $25.14 \mathrm{~nm}$. Images were recorded using line averaging and a dwell time of $200 \mathrm{~ns}$. 


\section{FIB-SEM data processing and 3D image reconstruction}

The resulting SE images were automatically aligned using the Fourier shift theorem for detecting the translational shift in the frequency domain and vertical stripes arising from the waterfall effect by FIB milling were removed by Fourier filtering. Total variation denoising was performed by applying the Chambolle algorithm in 3D mode. BSE images were processed using Dragonfly 4.1

(Object Research Systems, Montreal, Canada). Images were automatically aligned using the sum of square differences (SSD). Denoising was performed by applying a Median filter in 3D. Segmentation of individual structures (plaque vesicles, cell nuclei, mitochondria and cilia) was performed using the Deep learning tool in Dragonfly. Ten images were segmented by hand and then used to train the algorithm of the Deep learning tool. Once the training was successful the complete data set was segmented using the algorithm and inaccuracies and mistakes were corrected manually.

\section{References}

1. B. P. Lee, P. B. Messersmith, J. N. Israelachvili, J. H. Waite, Mussel-inspired adhesives and coatings. Annu. Rev. Mater. Res. 41, 99-132 (2011).

2. E. Khare, N. Holten-Andersen, M. J. Buehler, Transition-metal coordinate bonds for bioinspired macromolecules with tunable mechanical properties. Nat. Rev. Mater. (2021), doi:10.1038/s41578-020-00270-z.

3. M. J. Harrington, A. Masic, N. Holten-Andersen, J. H. Waite, P. Fratzl, Iron-Clad Fibers: A Metal-Based Biological Strategy for Hard Flexible Coatings - Supplement. Science. 328, 216-220 (2010).

4. E. Degtyar, M. J. Harrington, Y. Politi, P. Fratzl, The Mechanical Role of Metal Ions in 
Biogenic Protein-Based Materials. Angew. Chemie - Int. Ed. 53 (2014), pp. 2-21.

5. C. N. Z. Schmitt, Y. Politi, A. Reinecke, M. J. Harrington, Role of Sacrificial ProteinMetal Bond Exchange in Mussel Byssal Thread Self-Healing. Biomacromolecules. 16, 2852-2861 (2015).

6. C. Li, J. Zuo, Self-Healing Polymers Based on Coordination Bonds. Adv. Mater. 32, 1903762 (2019).

7. D. G. DeMartini, J. M. Errico, S. Sjoestroem, A. Fenster, J. H. Waite, A cohort of new adhesive proteins identified from transcriptomic analysis of mussel foot glands. J. R. Soc. Interface. 14 (2017), doi:10.1098/rsif.2017.0151.

8. J. H. Waite, Mussel adhesion - essential footwork. J. Exp. Biol. 220, 517-530 (2017).

9. T. Priemel, E. Degtyar, M. N. Dean, M. J. Harrington, Rapid self-assembly of complex biomolecular architectures during mussel byssus biofabrication. Nat. Commun. 8, 14539 (2017).

10. D. S. Hwang, H. Zeng, A. Masic, M. J. Harrington, J. N. Israelachvili, J. H. Waite, Protein- and metal-dependent interactions of a prominent protein in mussel adhesive plaques. J. Biol. Chem. 285, 25850-25858 (2010).

11. C. N. Z. Schmitt, A. Winter, L. Bertinetti, A. Masic, P. Strauch, M. J. Harrington, Mechanical homeostasis of a DOPA-enriched biological coating from mussels in response to metal variation. J. R. Soc. Interface. 12, 20150466 (2015).

12. T. Priemel, R. Palia, M. Babych, C. J. Thibodeaux, S. Bourgault, M. J. Harrington, Compartmentalized processing of catechols during mussel byssus fabrication determines the destiny of DOPA. Proc. Natl. Acad. Sci. 117, 7613-7621 (2020).

13. F. Jehle, E. Macías-Sánchez, P. Fratzl, L. Bertinetti, M. J. Harrington, Hierarchically- 
structured metalloprotein composite coatings biofabricated from co-existing condensed liquid phases. Nat. Commun. 11, 1-9 (2020).

14. A. Tamarin, P. J. Keller, An ultrastructutal study of the byssal thread forming system in Mytilus. J. Ultrasructure Res. 40, 401-416 (1972).

15. E. Filippidi, D. G. DeMartini, P. Malo de Molina, E. W. Danner, J. Kim, M. E. Helgeson, J. H. Waite, M. T. Valentine, The microscopic network structure of mussel ( Mytilus ) adhesive plaques. J. R. Soc. Interface. 12, 20150827 (2015).

16. A. Tamarin, P. Lewis, J. Askey, The structure and formation of the byssus attachment plaque in Mytilus. J. Morphol. 149, 199-222 (1976).

17. T. Ueki, N. Yamaguchi, Romaidi, Y. Isago, H. Tanahashi, Vanadium accumulation in ascidians: A system overview. Coord. Chem. Rev. 301-302, 300-308 (2015).

18. T. Ueki, T. K. Adi, Mechanism of vanadium accumulation and possible function of vanadium in underwater adhesion in ascidians. AIP Conf. Proc. 2120 (2019), doi:10.1063/1.5115602.

19. A. E. Ivliev, P. A. C. 't Hoen, W. M. C. van Roon-Mom, D. J. M. Peters, M. G. Sergeeva, Exploring the Transcriptome of Ciliated Cells Using In Silico Dissection of Human Tissues. PLoS One. 7, e35618 (2012).

20. Z. Movasaghi, S. Rehman, I. U. Rehman, Raman Spectroscopy of Biological Tissues. Appl. Spectrosc. Rev. 42, 493-541 (2007).

21. C. Kallepitis, M. S. Bergholt, M. M. Mazo, V. Leonardo, S. C. Skaalure, S. A. Maynard, M. M. Stevens, Quantitative volumetric Raman imaging of three dimensional cell cultures. Nat. Commun. 8, 14843 (2017).

22. M. Okada, N. I. Smith, A. F. Palonpon, H. Endo, S. Kawata, M. Sodeoka, K. Fujita, 
Label-free Raman observation of cytochrome c dynamics during apoptosis. Proc. Natl. Acad. Sci. U. S. A. 109, 28-32 (2012).

23. K. Hamada, K. Fujita, N. I. Smith, M. Kobayashi, Y. Inouye, S. Kawata, Raman microscopy for dynamic molecular imaging of living cells. J. Biomed. Opt. 13, 044027 (2008).

24. I. Michaud Soret, K. K. Andersson, L. Que, Resonance Raman studies of catecholate and phenolate complexes of recombinant human tyrosine hydroxylase. Biochemistry. 34, 5504-5510 (1995).

25. G. Azizi, M. Akodad, M. Baghour, M. Layachi, A. Moumen, The use of Mytilus spp. mussels as bioindicators of heavy metal pollution in the coastal environment. A review. $J$. Mater. Environ. Sci. 9, 1170-1181 (2018).

26. J.-C. Amiard, R. Journel, H. Bacheley, Influence of field and experimental exposure of mussels (Mytilus sp.) to nickel and vanadium on metallothionein concentration. Comp. Biochem. Physiol. Part C Toxicol. Pharmacol. 147, 378-385 (2008).

27. S. Nasrazadani, S. Hassani, in Handbook of Materials Failure Analysis with Case Studies from the Oil and Gas Industry (Elsevier, 2016; http://dx.doi.org/10.1016/B978-0-08100117-2.00010-8), pp. 39-54.

28. G. Danscher, Histochemical Demonstration of Heavy Metals A Revised Version of the Sulphide Silver Method Suitable for both Light and Electronmicroscopy. Histochemistry. 71, 1-16 (1981).

29. G. Danscher, M. Stoltenberg, Silver enhancement of quantum dots resulting from (1) metabolism of toxic metals in animals and humans, (2) in vivo, in vitro and immersion created zinc-sulphur/zinc-selenium nanocrystals, (3) metal ions liberated from metal 
implants and particles. Prog. Histochem. Cytochem. 41, 57-139 (2006).

30. F. D. Hardcastle, I. E. Wachs, Determination of molybdenum-oxygen bond distances and bond orders by Raman spectroscopy. J. Raman Spectrosc. 21, 683-691 (1990).

31. E. C. Theil, K. N. Raymond, in Bioinorganic chemistry (Mill Valley, California: University Science Books, 1994), pp. 1-35.

32. J. Costa Pessoa, E. Garribba, M. F. A. Santos, T. Santos-Silva, Vanadium and proteins: Uptake, transport, structure, activity and function. Coord. Chem. Rev. 301-302, 49-86 (2015).

33. I. Marigómez, M. Soto, M. P. Cajaraville, E. Angulo, L. Giamberini, Cellular and subcellular distribution of metals in molluscs. Microsc. Res. Tech. 56, 358-392 (2002).

34. T. L. Coombs, P. J. Keller, Mytilus byssal threads as an environmental marker for metals. Aquat. Toxicol. 1, 291-300 (1981).

35. J.-C. Amiard, H. Bacheley, A.-L. Barillé, L. Barillé, A. Geffard, N. Himery, Temporal changes in nickel and vanadium concentrations and in condition index and metallothionein levels in three species of molluscs following the "Erika" oil spill. Aquat. Living Resour. 17, 281-288 (2004).

36. S. G. George, B. J. S. Pirie, T. L. Coombs, The kinetics of accumulation and excretion of ferric hydroxide in Mytilus edulis (I.) and its distribution in the tissues. J. Exp. Mar. Bio. Ecol. 23, 71-84 (1976).

37. B. E. Brown, The form and function of metal-containing "granules" in invertebrate tissues. Biol. Rev. 57, 621-667 (1982).

38. S. G. George, T. L. Coombs, B. J. S. Pirie, Characterization of metal-containing granules from the kidney of the common mussel, Mytilus edulis. Biochim. Biophys. Acta-Gen. 
Subj. 716, 61-71 (1982).

39. N. Holten-Andersen, A. Jaishankar, M. J. Harrington, D. E. Fullenkamp, G. Dimarco, L. He, G. H. McKinley, P. B. Messersmith, K. Y. C. Lee, Metal-coordination: Using one of nature's tricks to control soft material mechanics. J. Mater. Chem. B. 2, 2467-2472 (2014).

40. N. Holten-Andersen, M. J. Harrington, H. Birkedal, B. P. Lee, P. B. Messersmith, K. Y. C. Lee, J. H. Waite, pH-induced metal-ligand cross-links inspired by mussel yield selfhealing polymer networks with near-covalent elastic moduli. Proc. Natl. Acad. Sci. U. S. A. 108, 2651-2655 (2011).

41. D. E. Fullenkamp, D. G. Barrett, D. R. Miller, J. W. Kurutz, P. B. Messersmith, pHdependent cross-linking of catechols through oxidation via Fe3+ and potential implications for mussel adhesion. Rsc $A d v .4$, 25127-25134 (2014).

42. P. Sharma, A. B. Jha, R. S. Dubey, M. Pessarakli, Reactive Oxygen Species, Oxidative Damage, and Antioxidative Defense Mechanism in Plants under Stressful Conditions. $J$. Bot. 2012, 1-26 (2012).

43. D. C. Crans, J. J. Smee, E. Gaidamauskas, L. Yang, The Chemistry and Biochemistry of Vanadium and the Biological Activities Exerted by Vanadium Compounds. Chem. Rev. 104, 849-902 (2004).

44. M. J. Sever, J. J. Wilker, Visible absorption spectra of metal-catecholate and metaltironate complexes. Dalt. Trans., 1061-1072 (2004).

45. A. Butler, C. J. Carrano, Coordination chemistry of vanadium in biological systems. Coord. Chem. Rev. 109, 61-105 (1991).

46. D. Rehder, The role of vanadium in biology. Metallomics. 7, 730-742 (2015). 
47. M. Henze, Untersuchungen über das Blut der Ascidien. I. Mitteilung. Die Vanadiumverbindung der Blutkörperchen. Hoppe-Seyler s Zeitschrift für Physiol. Chemie. 72, 494-501 (1911).

48. H. Michibata, The Mechanism of Accumulation of Vanadium by Ascidians: Some Progress towards an Understanding of this Unusual Phenomenon. Zoolog. Sci. 13, 489502 (1996).

49. T. B. Karpishin, T. D. P. Stack, K. N. Raymond, Octahedral vs Trigonal Prismatic Geometry in a Series of Catechol Macrobicyclic Ligand-Metal Complexes. J. Am. Chem. Soc. 115, 182-192 (1993).

50. S. A. Adediran, R. F. Pratt, Inhibition of Serine $\beta$-Lactamases by Vanadate-Catechol Complexes. Biochemistry. 47, 9467-9474 (2008).

51. N. A. Hamada, C. Gilpin, J. J. Wilker, Availability of Environmental Iron Influences the Performance of Biological Adhesives Produced by Blue Mussels. Environ. Sci. Technol. 54, 10254-10260 (2020).

52. N. R. M. Rodriguez, S. Das, Y. Kaufman, J. N. Israelachvili, J. H. Waite, Interfacial pH during mussel adhesive plaque formation. Biofouling. 31, 221-227 (2015).

53. J. H. Bernstein, E. Filippidi, J. Herbert Waite, M. T. Valentine, Effects of sea water pH on marine mussel plaque maturation. Soft Matter. 16, 9339-9346 (2020).

54. F. Timm, Zur Histochemie der Schwermetalle Das Sulfid-Silberverfahren*. Dtsch. Zeitschrift für Gerichtl. Medizin. 46, 706-711 (1958).

55. T. Lachmann, G. van der Snickt, M. Haschke, I. Mantouvalou, Combined 1D, 2D and 3D micro-XRF techniques for the analysis of illuminated manuscripts. J. Anal. At. Spectrom. 31, 1989-1997 (2016). 